\title{
Case Report: Generalized Mutual Information (GMI) Analysis of Sensory Motor Rhythm in a Subject Affected by Facioscapulohumeral Muscular Dystrophy after Ken Ware Treatment
}

\author{
Ken Ware1, Elio Conte ${ }^{2,3}$, Riccardo Marvulli ${ }^{2,4}$, Giancarlo Ianieri ${ }^{2,4}$, Marisa Megna ${ }^{2,4}$, \\ Enrico Pierangeli2, Sergio Conte ${ }^{2}$, Leonardo Mendolicchio' ${ }^{2}$, Flavia Pellegrino ${ }^{2}$ \\ ${ }^{1}$ The International Neurophysics Functional Performance Institute, Robina, Australia \\ ${ }^{2}$ School of Advanced International Studies on Applied Theoretical and Non Linear Methodologies of Physics, \\ Bari, Italy \\ ${ }^{3}$ Department of Basic Sciences, Neuroscience and Sense Organs, University of Bari Aldo Moro, Bari, Italy \\ ${ }^{4}$ Department of Physical Medicine and Rehabilitation, University of Bari Aldo Moro, Bari, Italy \\ Email: elio.conte@fastwebnet.it
}

Received 12 February 2015; accepted 27 March 2015; published 31 March 2015

Copyright (C) 2015 by authors and Scientific Research Publishing Inc.

This work is licensed under the Creative Commons Attribution International License (CC BY).

http://creativecommons.org/licenses/by/4.0/

(c) (i) Open Access

\begin{abstract}
In this case report we study the dynamics of the SMR band in a subject affected from Facioscapulohumeral Muscular Dystrophy and subjected to Ken Ware Neuro Physics treatment. We use the Generalized Mutual Information (GMI) to analyze in detail the SMR band at rest during the treatment. Brain dynamics responds to a chaotic-deterministic regime with a complex behaviour that constantly self-rearranges and self-organizes such dynamics in function of the outside requirements. We demonstrate that the SMR chaotic dynamics responds directly to such regime and that also decreasing in EEG during muscular activity really increases its ability of self-arrangement and self-organization in brain. The proposed novel method of the GMI is arranged by us so that it may be used in several cases of clinical interest. In the case of muscular dystrophy here examined, GMI enables us to quantify with accuracy the improvement that the subject realizes during such treatment.
\end{abstract}

\section{Keywords}

Ken Ware Neuro Physics Treatment, SMR Band, Generalized Mutual Information, Chaotic Brain Dynamics 


\section{Introduction}

In human EEG, primary sensory or motor cortical areas typically exhibit rhythmic activity at a frequency that may be characterized covering approximately the band $8-12 \mathrm{~Hz}$. It may be observed when they are not processing sensory information or producing motor output. This kind of brain activity has been identified as mu rhythm, and is retained that it is produced as a consequence of interactions between the thalamus and the cortex. Results have demonstrated that mu rhythm activity actually covers a variety of different rhythms that are distinguished by location, frequency, and/or relationship to concurrent sensory input or motor output. It is a very important band not only for understanding basic brain dynamics but also because, as we know, a number of results indicate that mu rhythm activity can be a good carrier for BCI-based communication. These rhythms are associated with those cortical areas that are most directly connected to the brain's normal motor output channels. Among the basic features of such rhythm, it results of particular interest that the movement or still also the simple preparation to the movement is typically accompanied by a decrease in mu activity over sensorimotor cortex, particularly contralateral to the movement. Very important studies have been conducted and we quote in particular here those of Pfurtscheller. We invite the reader to consider his basic work and all the other results indicated in the reference of his work. The decrease in mu activity has been called "event-related desynchroni-zation" or ERD to be distinguished from event-related synchronization (ERS) occurring in the post-movement period and with relaxation [1]. Furthermore, and most relevant for BCI applications, ERD and ERS have been observed also with motor imagery, that is to say, when we imagine movement and thus when we do not require actual movement [1]. We outline again that, since they can occur independent of activity in the brain's normal output channels of peripheral nerves and muscles, they can serve as the basis for a BCI.

In order to give an exhaustive representation of the functional and dynamical regime, we may report here in Figure 1 the excellent images given in $\mathrm{http}: / / \mathrm{www}$.snipview.com/q/Sensorimotor\%20 rhythm.

According to $h$ ttp://www.snipview.com/q/Sensorimotor\%20rhythm, we have in A, and B sections the topographical distribution on the scalp of the difference (measured as $r^{2}$ (the proportion of the single-trial variance that is due to the task)), calculated for actual (A) and imagined (B) right-hand movements vs. at rest for a $3 \mathrm{~Hz}$ band centered at $12 \mathrm{~Hz}$. In $\mathrm{C}$ we have an example voltage spectra for a different subject and a location over left sensorimotor cortex (i.e. C3) for comparing rest (dashed line) and imagery (solid line).

In D one has the corresponding $r^{2}$ spectrum for imagery vs. at rest. Signal modulation is focused over sensorimotor cortex and in the alpha- and beta-frequency bands associated with mu rhythm activity.

Studies about such brain dynamics electromagnetic activity started Sterman in 1966 (see in details the studies indicated in [2]-[5]). The author performed EEG recordings obtained during the elaboration of conditioned response in animals and disclosed two behaviorally specific EEG patterns. One was related to the learned suppression of the bar-press response during presentation of a tone, and the other to the reward following completion of a correct response (for details, see the original exposition of the experiment by this author). The EEG pattern

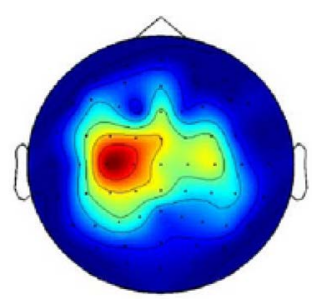

(a)

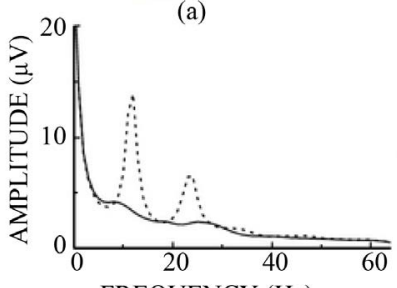

FREQUENCY $(\mathrm{Hz})$

(c)
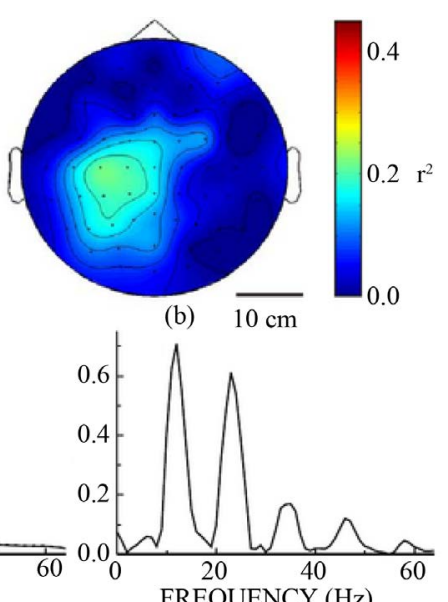

(d)

Figure 1. Reproducing the basic feature of the involved dynamics (http://www.snipview.com/q/Sensorimotor\%20rhythm). 
associated with the suppression of bar-pressing behavior in hungry animals was a $12-20 \mathrm{~Hz}$ rhythm localized to sensorimotor cortex. Because of this localization the authors labelled this the "Sensorimotor Rhythm," or SMR. This pattern was, indeed, very similar to the characteristic sensorimotor EEG spindle burst seen during sleep. This fact suggested that some aspect of underlying neurophysiology was common to these two behavioral states. The EEG pattern associated with food reward, on the other hand, was a $4-12 \mathrm{~Hz}$ rhythm localized to posteriordorsal (parietal) cortex. In the well-trained animal it was seen as soon as the feeder mechanism was activated and during food consumption. Because of this consistent association with primary and secondary reward components the term "Post-reinforcement Synchronization or PRS" was introduced. Similar activity was seen also during ad lib drinking, grooming, and drowsiness indicating some common physiological feature among these states as well.

These authors outline that these two patterns were the only clear instances of visually detectable rhythmic EEG activity observed in the alert cat. Further, they were distinguished by the fact that they emerged clearly from a background of non rhythmic, low voltage EEG activity. Although frequency analysis of the SMR showed a range of activity between 12 and $20 \mathrm{~Hz}$, peak activity for this rhythm was found at approximately $12-14 \mathrm{~Hz}$. Operant conditioning was thus initiated with an active signal detection filter centered at $13 \mathrm{~Hz}$, and set to activate the feeder device when criterion amplitude and duration triggered a relay [3] [4]. The authors found that cats could easily be trained to produce this rhythm voluntarily. Between 150 and 200 conditioned responses could be elicited prior to satiety, occurring at approximately $5-10 \mathrm{~s}$ intervals during a typical experimental session.

The behavior associated with this conditioned response was again the sustained suppression of motor activity. However, movement suppression was necessary but not sufficient for production of the rhythm. Careful studies of muscle activity changes associated with SMR training showed that each response was immediately preceded by a 4 - 6 s period of graded muscle tone reduction, at the end of which the SMR would appear [4] [5]. These changes in muscle tone occurred within the context of a sustained immobility.

We have given preliminarly such historical account in order to evidence the importance of SMR studies in muscular disfunctions.

Generally speaking we know that Duchenne (DMD) and Becker (BMD) muscular dystrophies are X-linked recessive disorders due to complete or partial loss of dystrophin protein. Myotonic dystrophy (DM) is a chronic and slowly progressive, autosomal-dominant, multisystem disease characterized by a wide spectrum of clinical findings and by marked intra familial and interfamilial clinical variability. There are two genetically distinct form of DM, myotonic dystrophy type 1 (DM1) and type 2 (DM2) with different clinical phenotypes. The most frequent form is DM1 and on the basis of clinical severity the disorder is divided into three groups: mild, classical, and congenital. DM1 is caused by a (CTG) expansion in the gene encoding Dystrophia-Myotonica-ProteinKinase (DMPK), a zinc finger protein. When transcribed into CUG-containing RNA, mutant transcripts aggregate as nuclear foci that sequester RNA-binding proteins, resulting in a spliceopathy of down stream effector proteins (troponin, insulin receptor, etc.). DM1 may be considered as a toxic RNA disease [6] [7]. Facio Scapulo Humeral Muscular Dystrophy (FSHD) is the third most frequent form of muscular dystrophy. Emery-Dreifuss Muscular Dystrophy (EDMD) is genetically heterogeneous since it may be caused by a mutation in the STA gene encoding emerin (X-linkedinheritance) or as AD trait determined by mutations in the LMNA gene encodinglamin A/C.EDMD is clinically characterized by a slow progressive muscular dystrophy. Miyoshi muscular dystrophy (MMD) is caused by mutation in the gene encoding the protein dysferlin. For a complete review and quotation of the papers of all the authors that performed such detailed studies, see [8].

The present paper is a case report. We give the results of SMR analysis in a subject affected by Facioscapulohumeral Muscular Dystrophy. We perform the analysis comparing the results before and after the treatment performed by Ken Ware. From a clinical point of view we are interested to examine the results of the treatment. From a methodological point of view we are interested to apply an appropriate methodology of analysis that of course could be applied as general method in future applications. Let us sketch the problem in detail from a methodological view point. It is well known that EMG signal is a measure of the electrical activity associated with the contraction of a muscle. Currently, the EMG signal is explored by different measures or signal features. Standard techniques include time-domain features such as zero crossing and root mean square (RMS), stochastic features as autoregressive model coefficients, cepstral coefficients, mean frequency and median frequency and other techniques as wavelet coefficients. When examining the question in detail we have to acknowledge that the EMG signal originates actually from a strong non linear combination of similar distributions due to the sin- 
gle fiber action potential. Consequently, the previous mentioned methods, being all based on linear elaboration, cannot give accurate results since instead an analysis of the non linear and thus possibly chaotic systems is required [9]-[15]. In the present paper we use the method of the Generalized Mutual Information (GMI) that in fact was introduced years ago [16] just to account for the flow of information and dependencies in times series and between time series looking in detail to the non linear, possibly chaotic, acting mechanisms in time dynamics.

\section{Materials and Methods}

\section{The Treatment [17]}

The patient is a 60 -year-old male. He had been suffering for more than 30 years from the rare form of Muscular Dystrophy, Facioscapulohumeral Muscular Dystrophy (FSHD). (FSHD) is a genetic muscle disorder in which the muscles of the face, shoulder blades and upper arms are among the most affected. EEG, ECG, EMG were all recorded simultaneously using BioRadio 150 technology. EEG was recorded in channel 8 with electrodes were placed at C3-F3 of the left hemisphere. ECG was recorded in Channel 7 with electrodes placed at V1 and V2. An appropriate software by BioPac sytem enabled us to obtain the relative R-R intervals for HRV analysis. The EMG recording was performed on six channels. In Channel 1 the electrodes were placed lengthwise on right trapeze. In channel 2 the electrodes were placed lengthwise on left trapeze. The reason to select such regions is that the trapezes are highly reactive to activation of the sympathetic nervous system (SNS). Actually, there is a significant increase in tone in this region when the subject becomes anxious or fearful. Any significant differences between tone and synchronization between the left and right trapeze are indicative of undesirable hemisphere dominance whereas dominantly stable bilateral synchronization is observed in healthy men and women. The EMG recording in channel 3 was realized with electrodes placed on the right abdominals, laterally adjacent to the navel. The EMG recording in channel 4 was realized with electrodes placed on the left abdominals, laterally adjacent to the navel. The EMG recording in Channel 5 was realized with electrodes placed lengthwise on the adductors of the lower right limb (leg) and, finally, the EMG recording in channel 6 was realized using electrodes placed lengthwise on the adductors of the lower left limb (leg). The placement of the EMG electrodes on the Trapezes, Abdominals and Adductors, enabled us to monitor the systems overall responses to the mild stimulus provided by the resistance exercise, when initiated by the patient. However the patient was recorded for 5 minutes at rest before therapy began. This initially measurement was deemed to represent the diseased state of the patients system at rest. For comparison, it is observed in the streaming of the raw data, of healthy young adults at rest, that there is a lot of synergy; frequency and amplitude-displaying fractal variability over time between all of the the EMG channels, when placed at the same locations described above. This is indicative of a well-distributed "flow" of energy and information throughout the system. It is also observed that when healthy young adults are asked to purposely tense their trapezes mirroring the effects of activation of the sympathetic nervous system on this region, that this perturbation results in the streaming raw data relative to this region, displaying massive frequency with low amplitude and no variability over time. The purposeful tensing of the trapeze also creates an immediate gross loss of "flow" and synchronization between the other channels; observed as an immediate loss of amplitude and fractal variability. The excessive tension in the trapezes significantly interferes with healthy "open system" sensory motor processing. It is commonly observed in patients with chronic complex diseases and mental disorders, that there has been long-term hyper-vigilance in the trapeze regions, which is indicative of long term, over activation of the (SNS) and parasympathetic nervous system dysfunction. It is common that these patients will report that they have been under long term, high order stress and anxiety The exercise equipment used is specialized exercise equipment supplied to the International Neuro Physics Functional Performance Institute by "Synergy Fitness"

(http://synergyfitness.com.au/gallery/15/Synergy_4_Pin_Loaded)

The Ken Ware Neuro Physics Treatment (NPT) that we have considered involves no physical manipulation of the treated individual. Individuals perform the exercises on their own with specialize instructive coaching support. The exercises are not tailored to particular presenting conditions: the same approach is used regardless of age, injury, medical history, or disorder. Treatment is a phased process. Session frequency during the brief initial treatment period may vary from daily, to alternating days, to several days apart, depending upon the general condition of the subject. In our case the patient did four days of consecutive intensive therapy, with each session 
lasting 2 hours. Selections of exercise equipment are also dependent upon a patient's ability or disability. Usually early phase equipment is the leg press (which may be substituted with the seated leg curl), lateral pull down, and pectoral exercise, the pec dec. Each exercise machine loaded with very light weight, mostly representative of the equipment's minimum lifting loads. Most movements have only two degrees of freedom $(d f)$ (up-down or forward-back). Only one machine used in the early phase of treatment has $4 d f$, namely the pec dec. The initial treatment in our case, involved four models of variable resistance exercise equipment, which were selected in consideration to the level of ability of the patient. No stretching or warm-up exercises are permitted to preserve a patient's system normal resting state, innocent of perturbations until the (NPT) starts. The initial assessment session(s) evidences the patient's chronic system condition and how it interacts with and evaluates its environments in general. The specific activities and equipment used at the beginning of treatment are expanded after initial sessions' effects area assessed and systematically modulated as necessary, through the unique applications and qualities of NPT. In the next treatment phase, additional exercises that are slightly more complex to perform, but with still very lightly weighted, are added to the patients program, again depending on ability or disability. Some of these pieces of equipment have many $(d f)$; the handles are attached to cables that can move in any direction. The patients are at this time (re) introduced to so-called "cardio" exercises (this nuisance term promoted by the exercise industry un-holistically implies the cardiovascular system is separate, requiring its own form of exercise). In the reinforcement and maintenance phase of the program, additional equipment with prescribed repetitions and weights is introduced. Individuals in treatment perform the following activities with trained coaching support). The basic features of the treatment may be summarized as follows: a) Learn to relax physically, mentally, and emotionally; b) Adjust position for proper contact points and symmetrical balance on equipment; c) Maintain up right posture; d) close eyes (recommended); e) Perform ultra-slow movements; f) form and operationalize the intention to distribute effort and energy throughout the body (pec dec), The initial treatment in our case, involved four models of variable resistance exercise equipment, which were selected in consideration to the level of ability of the patient. No stretching or warm-up exercises are permitted to preserve a patient's system normal resting state, innocent of perturbations until the (NPT) starts; g) exert executive control in order to maintain ultra-slow speed and balance, engage the processes of encountering resistance per coaching instructions (e.g. to pause, stop, restart, modulate), to allow and relax into the varying intensities of random, chaotic, or rhythmic physical movements, and finally, when instructed to do so, terminate various movements in a calm fashion. In addition, to respond to instructions (e.g. adjust posture, relax, open eyes to observe and rebalance positioning, or respond in a particular way to body's movement or emotion) and after early treatment phases, practice control to maintain poised balance between slipping into the chaotic dynamics and not doing so when practicing the exercises. Ultra-slow movements are performed during the initial stages of the therapy. The slow speed's role for the central nervous system is analogous to a good mechanic listening to an engine's idling state: at slow speeds, imbalances are detectible. At high speeds, rapid revolutions mask the symptoms of underlying imbalances. Daily human life tends to run at high throttle. In contrast, by moving a light load very slowly with few $d f$ on each piece of equipment, the nervous system can detect and become sensitive to its own initial conditions. This is prerequisite for the system to then adjust and monitor itself. Its next behaviours, during and after each therapy session, are dependent on those progressively-changing initial conditions. Patients with slumping posture or muscle tension are coached to straighten, relax and practice executive control to correct them. If a light involuntary dynamical motor movement is observed in a limb or any smaller remote region during a repetition of a movement, the patient is asked to suspend activity at that position to permit the dynamic response to surface and evolve. Such mild indicators are the system's first hint that a person is beginning to remove physical-emotional restraints and unbridle their system. To cease movement in an unconstrained, relaxed poise sets the conditions for a full, or fuller, range of system dynamics to release. Often, just before such release, the person reports that the mild weight feels very difficult to move, usually expressing anxiety about it. It feels harder to move even light weight at ultra-slow speeds because it costs more effort and thus energy, because the sensory motor system is enable to elaborate on the environmental stimulus. This overview implies the method's key therapeutic premises. The overarching premise is that certain conditions evoke transitions into and out of system-controlled chaos, requisite for the system to reorganize itself to healthier balance.

The patient presented on the 13th of October, 2014. His head was dropped into his right shoulder. He had significant drooping of the mouth and compromised normal facial tones and expressions. His right trapeze was excessively tensed (hyper-vigilant), whilst the left trapeze was comparatively very low in tone. He was not able to 
hold his head up straight for any period of time for several years. He could not close either of his hands to make a fist for several years. He was not able to clap his hands together for several years. His right foot was significantly pronated with long-term spasticity. He had poor control over his abdominal/lumber stability process. The patient began an assisted walking program with his physiotherapists on the 8th of August 2104. He was progressing slowly until midway through September 2014, when he was infected with a virus. The virus weakened him significantly and set the walking program right back. He began his walking program again post (NPT) on the 22nd October 2014. His progress significantly increased 4 fold in one month to the 25th November 2014-opposed to an $80 \%$ increase in the first month of the walking program pre (NPT). The valuable benefits the patient obtained in 4 days from (NPT) may be reassumed as follows: 1) Able to hold his head up straight and even on his shoulders; 2) Able to smile and form other facial expressions that had been lost for many years; 3) Full relaxation of spasticity in right foot; 3) Able to close hands (fingers onto palm of hand); 4) Able to clap hands together (patient reported that he had not been able to do this for 7 years before (NPT)); 5) Able to support himself significantly more via his abdominal/lumber stability process. Over the 4 days of intensive (NPT), this muscular dystrophic patient obtained substantial benefits from the therapy.

Let us explain the essence of our method. As previously said we recorded EEG (C3-F3) and EMG on trapezes (left and right) signed at channels (Ch1 and Ch2) sampling at a frequency of $960 \mathrm{~Hz}$. In relation to the EEG analysis we performed first of all standard Fast Fourier Transform obtaining the SMR band that we selected in the range $13-15 \mathrm{~Hz}$. We used also young subjects for controls. As second step we used the previously mentioned GMI analysis. Consider a stationary time series $\left\{X_{t}\right\}$, where $X_{t}$, is a real-valued random variable representing the observation $x_{t}$ made at time $t$. The series is assumed to be time-discrete as actually is in our case.

Let $P_{m}$ denote the probability that $X_{t}=x(m)$. For a given time lag $\tau, X_{t}$ and $X_{t+\tau}$ are said to be statistically independent if $p_{m n}(\tau)=p_{m} p_{n}$ for all $m, n=1,2,3, \cdots, k$ where $p_{m n}(\tau)$ is the joint probability to $X_{t}=x(m)$ and $X_{t+\tau}=x(n)$.

The most common measure of statistical dependences in such a stationary time series is the autocorrelation function (ACF). However, the ACF measures only linear dependences, which are the only ones for very special distributions of $\left(X_{t}, X_{t+\tau}\right)$, e.g. jointly normal. It cannot be applied in the present measurements since brain EEG as well as EMG evidence strongly non linear basic time dynamics. In the cases of non linear, possibly chaotic, systems it becomes necessary to measure the mutual information $I(r)=H_{1}-\left(H_{2}(\tau)-H_{1}\right)$ using Shannon entropy. By using the generalized Rényi entropy Pompe was able to estimate the Generalized Mutual Information (GMI) $I_{D}^{2}(\tau)=H_{1}^{(2)}-\left(H_{D+1}^{(2)}(\tau)-H_{D}^{(2)}\right)$ that is able to estimate the dependency and the flow of information when non linear acting contributions are acting in the investigated time series. Let us explain now the essence of our methods. Given the recorded EEG we divided the whole file into groups of 60,000 points (considering that the sampling frequency is $960 \mathrm{~Hz}$, this is to say that we examined EEG dynamics in following time steps every 62.5 seconds) with time lag tau varying from zero to 200 . This is to say that we examined the minimum value of $4.80 \mathrm{~Hz}$ until to covering the whole bands that are of interest in EEG for our purpose. In particular the range enclosed between 64 and 74 tau covered the range of $13-15 \mathrm{~Hz}$ of SMR band. The aim was to examine the flow of information and thus the coupling, the statistical dependency and thus synchronization in brain dynamics every 62.5 seconds with particular consideration for the SMR band. The results of the application of the method are reassumed in the following Figures. The interpretation is as follows: every time we find a peak in GMI estimation, this is to say that here we have strong dependency, coupling of events in brain dynamics, higher flow of information, synchronization. It is important to outline here again that we are using a method estimating the presence of strong non linear-chaotic contributions.

In conclusion, let us sketch the essence of the method that we employed. We have recorded EEG in channel 8 and left and right trapezes mioelectric activity in channels $\mathrm{Ch} 1$ and $\mathrm{Ch} 2$. We have sampled at a frequency of 960 $\mathrm{Hz}$, and this is to say that each time lag tau is 1.0416 milliseconds. Let us call, as example, the EEG recorded activity by $X(t)$, the recorded Ch1 EMG activity by $Y(t)$ in Channel 1 (left trapeze) and $Z(t)$ in channel 2 (right trapeze). The essence of our research is now to evaluate the Generalized Mutual Information, GMI, and this is to say the flow of information, the dependency and thus, considering the corresponding frequencies, the coupling and the synchronization one time in $X(t)$ respect to $X(t+\tau)$ (in this manner we estimate GMI dependency between correlating events in the brain ) and one time, as it is the case of the following paper that we have in preparation, between $X(t)$ and $Y(t+\tau)$ and respectively between $X(t)$ and $Z(t+\tau)$, thus evaluating in this cross manner the functional dependence between brain and trapezes EMG. The essence of the 
analysis is that we perform an investigation based on the presence of non linear-chaotic mechanisms, one time in the brain and the other time in brain respect to left trapeze and, respectively, right trapeze.

\section{Results}

Let us look first of all to the result obtained for the controls using Fast Fourier Transform (FFT).

Let us examine the results that were obtained in Figure 2. It is the case of the controls. In abscissa we have indicated the sequences of file Ch8-a-b EEG recording every $15.624 \mathrm{sec}$. In ordinate we have the Power Spectral Density $\left(\mu \mathrm{V}^{2} / \mathrm{Hz}\right)$ in the SMR band $(13-15 \mathrm{~Hz})$. We have the results for the subject at rest and the same subject during an exercise so to activate movement.

The whole time of examination is $156.24 \mathrm{sec}$ fragmented in ten sections (Ch8-1-1, Ch8-1-2 ...). The bleu graph indicates the PSD of the SMR band at rest and we observe that we have a PSD ranging from 10 to 30 $\left(\mu \mathrm{V}^{2} / \mathrm{Hz}\right)$ as expected for a subject at rest in accord also with the basic results indicated in the literature evidenced in Figure 1. The red graph represents instead the behaviour of the SMR band for the subject under exercise. The corresponding values are about $2.5\left(\mu \mathrm{V}^{2} / \mathrm{Hz}\right)$ in satisfactory agreement with the dynamics of such brain rhythm that, as it is well known, tends to decrease drastically during the movement.

Let us examine now Figure 3 that relate instead the subject affected from Facioscapulohumeral Muscular Dystrophy. As previously said the patient had two section of (NPT) treatment that is indicated by Lat Pull 13 and Lat Pull 17. Therefore we compare two at rest conditions before treatment of Lat Pull 13 and after treatment of 17 respectively and, in addition, we have estimation of at rest against during the treatments.

Observing the results reported in Figure 3 we have that the subject starts with a PSD that is exceeding the range of the normal values by several orders. This subject, in fact, has values covering the range of 5000 to $95,000\left(\mu \mathrm{V}^{2} / \mathrm{Hz}\right)$ that is strongly exceeding the standard value of about $30\left(\mu \mathrm{V}^{2} / \mathrm{Hz}\right)$ found in the normal subject. The important feature to be outlined is that, after the treatment, (red graph) the subject recovers mainly rather normal values with only the two exceeding values in Ch 8-6 and Ch 8-7. Such exceeding values, however, are about $12,000-21,000\left(\mu \mathrm{V}^{2} / \mathrm{Hz}\right)$ evidencing a net improvement of the subject SMR brain dynamics after the treatment. In substance the starting pathological condition of the subject gives an SMR band with values of about $70.71-308 \mu \mathrm{V} / \mathrm{Hz}$. After the treatment such SMR band activity ranges from prevailing normal values to exceeding values identified only in the two time intervals $\mathrm{Ch} 8-6$ and $\mathrm{Ch} 8-7,109.54-144.49 \mu \mathrm{V} / \mathrm{Hz}$. The subject received an unquestionable improvement of his pathological condition during the treatment.

In Figure 4 we have also the analysis of the SMR band during the two sections of the treatment.

The estimation of the SMR band PSD during the two sections of the treatment evidences the net increased SMR activity during the two session of the treatment with greater values during the second session (lat pull 17) respect to the previous one (lat pull 13).

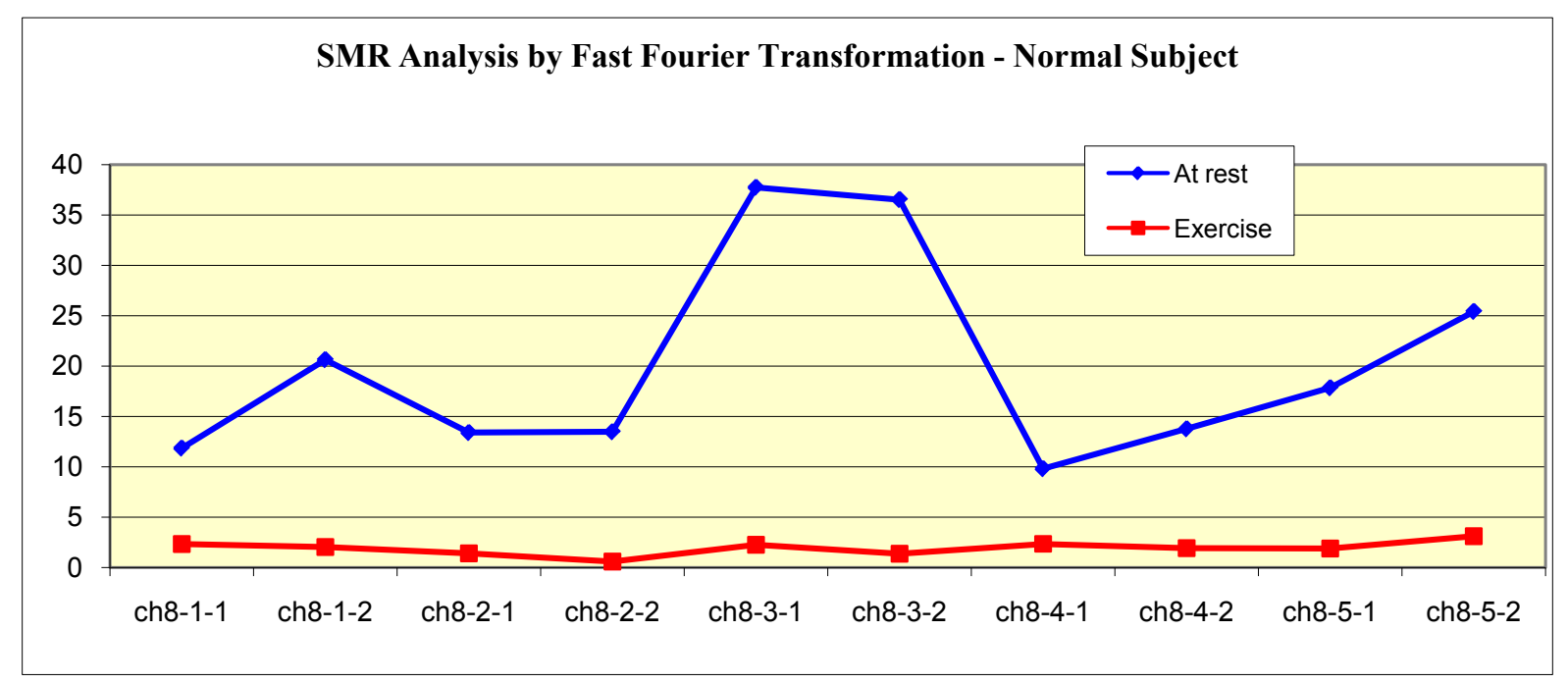

Figure 2. FFT in the SMR band of EEG in the control subject at rest (pre-exercise) and during an exercise (pec dec). 


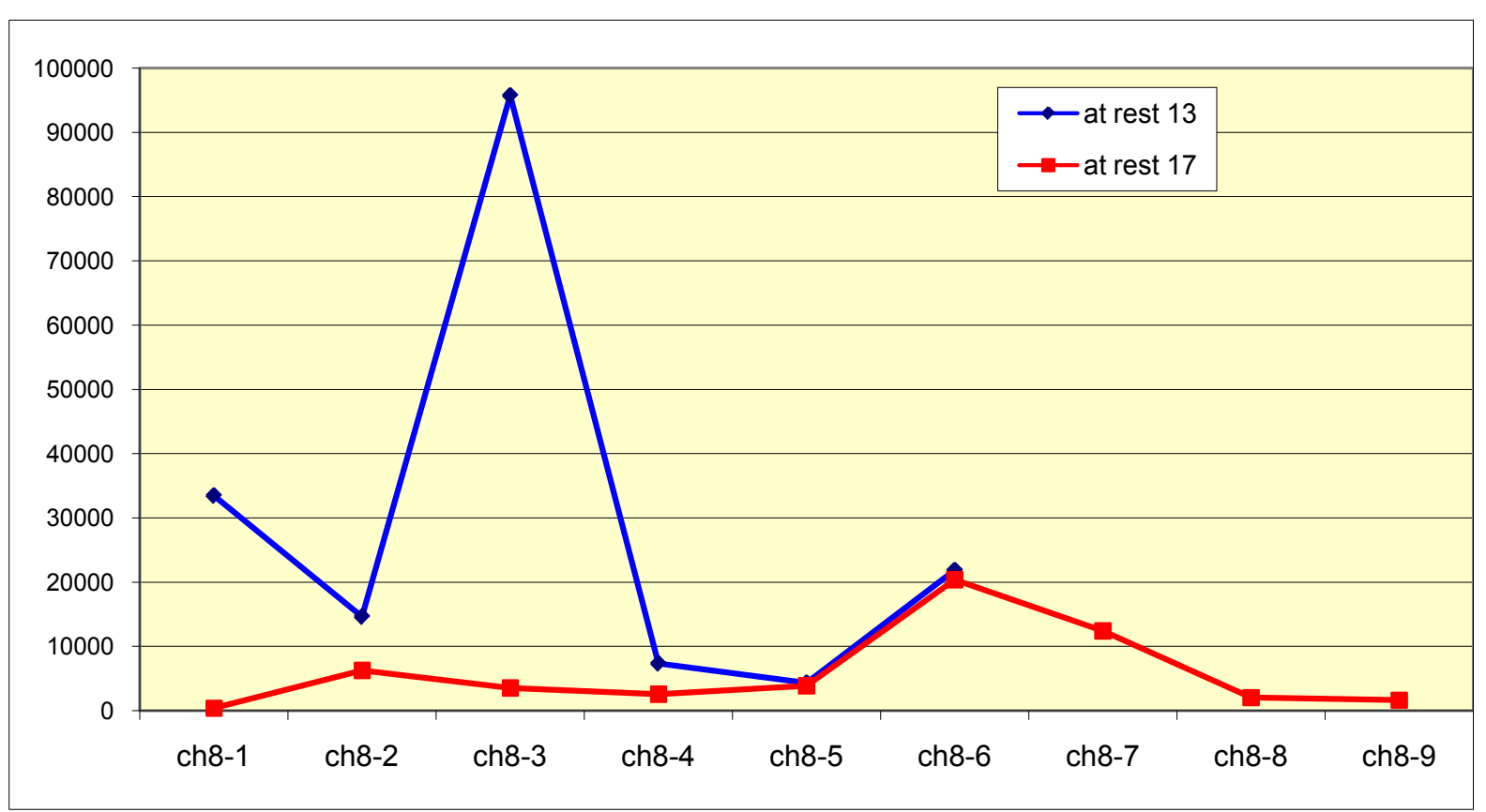

Figure 3. Analysis of SMR band in the two at rest conditions . Before the treatment (at rest 13) and after the treatment (at rest 17).

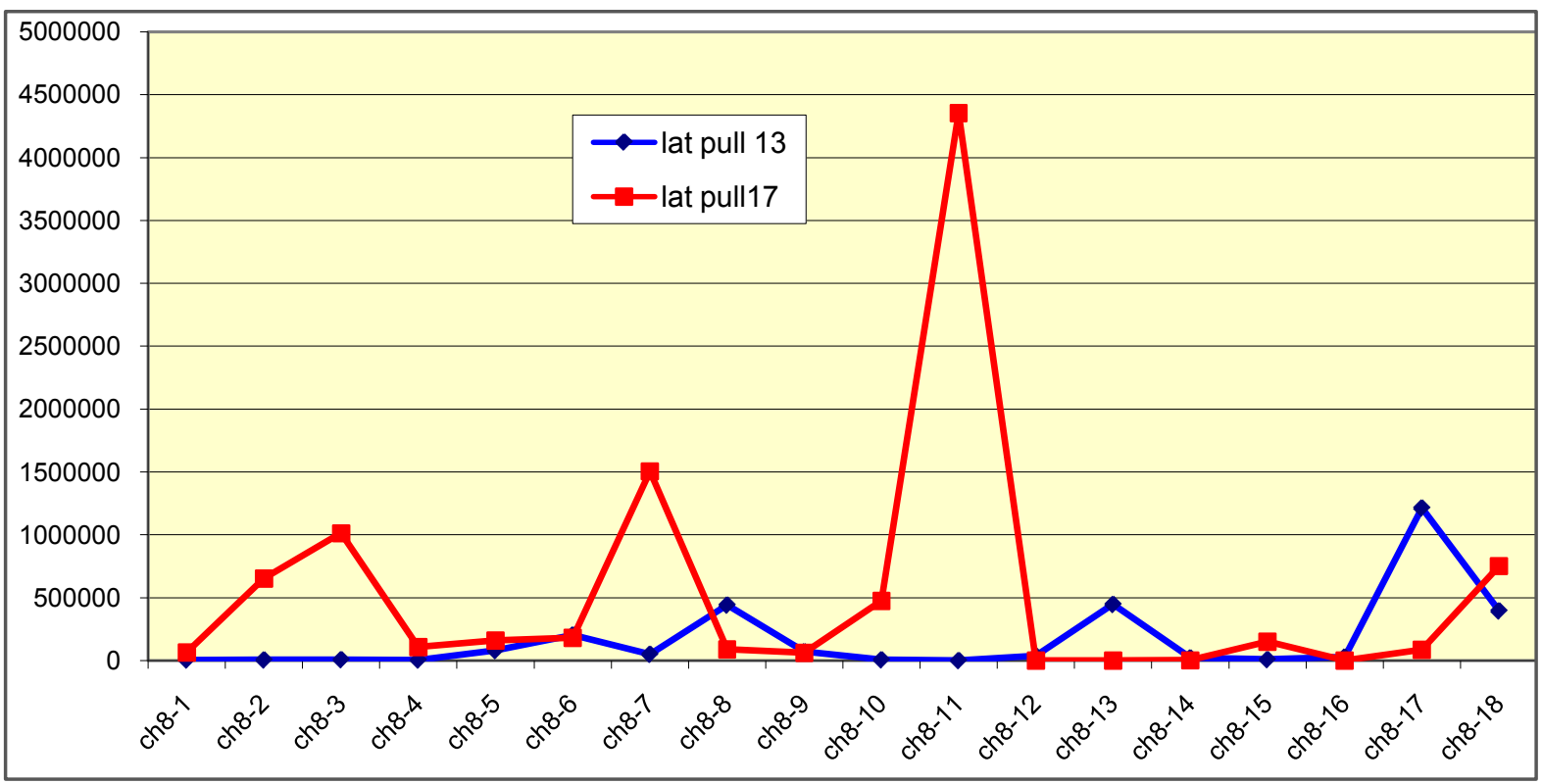

Figure 4. Estimation of the SMR band during the treatment.

From a neurological point of view we have to introduce the following considerations: as previously outlined, the SMR or $\mu$ band in humans originates from motor cortex and it is linked to motor neuron activity. In the subject under consideration, affected from Facioscapulohumeral Muscular Dystrophy, the activity of motor neurons has profound alterations that are due to the primitive muscular damage. Consequently the neurophysiological response that we record in the SMR band results to be totally different respect to normal subjects. This observation explains the reason because we obtain so increased values in the SMR band in the pathological case respect to the controls. After all, they continue to respond during the treatment giving a net recovery at rest after the treatment has been performed. 
We may now pass to examine the results obtained by application of the GMI method.

First of all let us examine the EEG-GMI results in the case of the normal subject at rest. The results are given in Figure 5.

In Figure 5 we have the results of the GMI analysis for the normal subject at rest. In the upper side we have GMI results for the lags ranging from 0 to 200 and thus covering all the brain rhythms. It is immediately evidenced the satisfactory agreement obtained in the coherence, coupling, synchronization in all the brain dynamics. The flow of information is expressed in Bit and the brain gives a constant value that is about 0.4 for all the fragments of recorded brain activity.

In the lower graph we have the GMI values for the SMR band only. Different fragments are examined and we have a constant value of GMI ranging from 1.760 to 1.786 Bit. Note also that we have always three peaks respectively to $64,68,72$ time lags corresponding respectively to $15 \mathrm{~Hz}, 14.118 \mathrm{~Hz}$ and $13.334 \mathrm{~Hz}$ (SMR band). The GMI value is constant about 0.42 Bit.

Let us examine the situation during the exercise. The results are given in Figure 6.
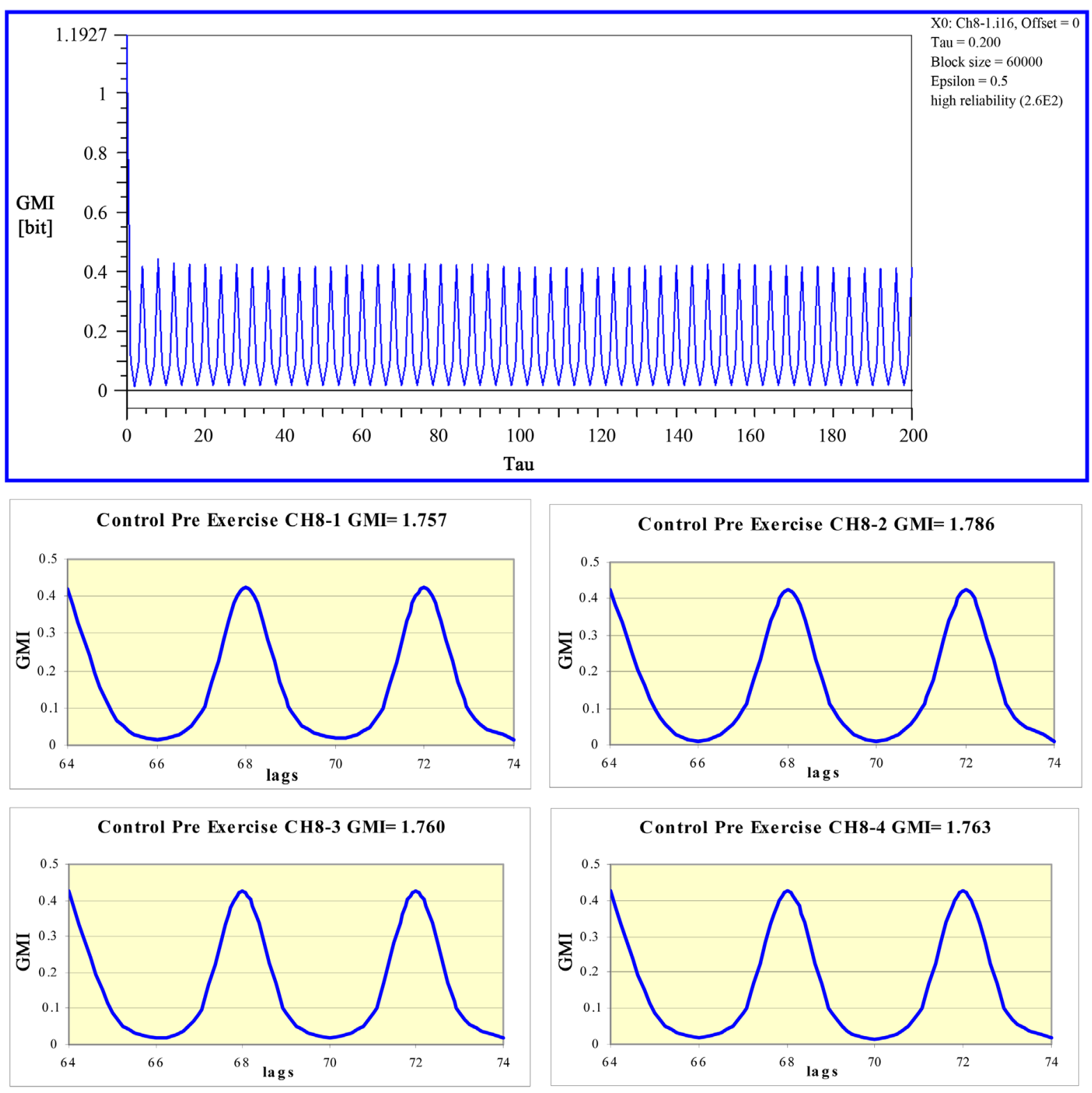

Figure 5. GMI analysis of the EEG in healthy subject at rest. 

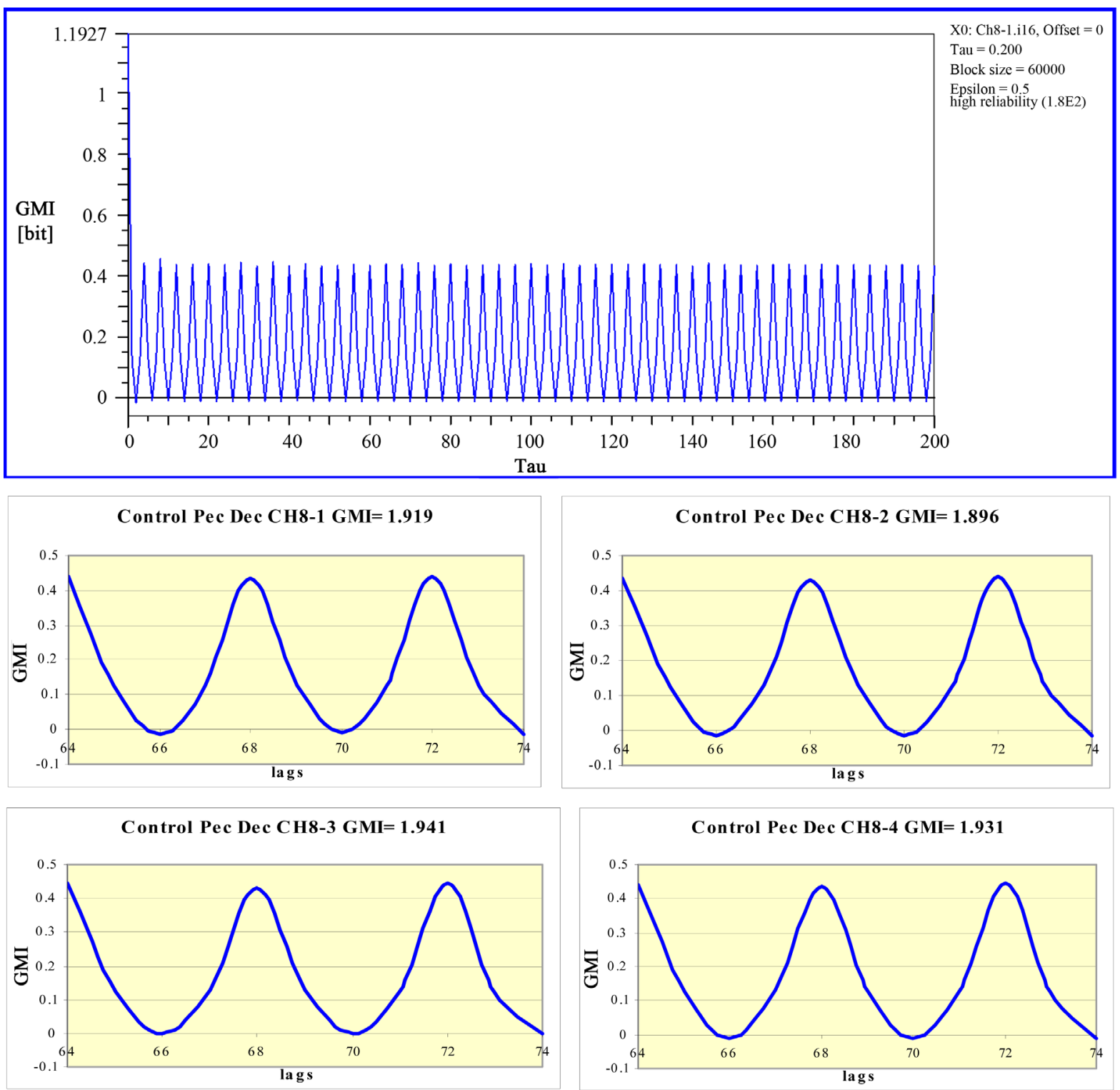

Figure 6. GMI analysis of the EEG in healthy subject during the exercise.

In Figure 6 we have the GMI results when the control subject is performing the exercise and thus he is in movement. In spite of the fact that in the EEG recording we observe an attenuation of the activity of the SMR band, by using appropriate methods of non linear chaotic analysis we discover that brain maintains its total coherence, dependency, coupling and synchronization in all the bands charactering its dynamics. In particular the movement induces a light modification of the flow of information passing the GMI from the previous value about to $0-42$ to 0.46 Bit. The analysis of the SMT band reveals that we have the conservation of the same peaks at $15 \mathrm{~Hz}, 14.118 \mathrm{~Hz}$ and $13.334 \mathrm{~Hz}$ (SMR band) but we have a significant increment in the final values of the GMI ranging this time from 1.896 to $1.941 \mathrm{Bit}$. In order to manage the movement, brain dynamics increases the flow of information, the dependency, the coupling, the synchronization, self-organization and self-arrangement and the particular advantage of our method is that it is available to quantify with accuracy such increased whole coherence in the SMR band. We have now a robust index, the GMI, which evaluates step by step how brain dynamics remains self-arranged and self-organized so that motor neurons may explain their activity with total synchronization.

We may now appreciate the sensitivity of our methodology examining the GMI results obtained for the sub- 
ject affected from Facioscapulohumeral Muscular Dystrophy. In Figure 7 we give the results of the subject at rest before starting the treatment.

In Figure 7 upper side we observe immediately that the subject no more has coherence, dependency, coupling and synchronization in all the brain bands as observed in the case of the healthy subject. The graph evidences some peaks immediately followed from an incoherent dynamics. The GMI values are decreased from $0.42 \mathrm{Bit}$ in the normal case to a value that oscillates between 0.05 and $0.25 \mathrm{Bit}$. We have a reduction of the index value oscillating between $50 \%$ and $90 \%$.

The GMI index, accounting finally for the non linear chaotic regime, gives detailed indication of the serious pathology of the subject.

It is of interest also the analysis of the SMR band (lower Figures). The standard configuration with peaks at $15 \mathrm{~Hz}, 14.118 \mathrm{~Hz}$ and $13.334 \mathrm{~Hz}$ (SMR band) frequently is missing. Spurious peaks appear at 14.556 and $13.717 \mathrm{~Hz}$ and the basic peak at $15 \mathrm{~Hz}$ is attenuated. The brain dynamics of the subject in the SMR band recov-
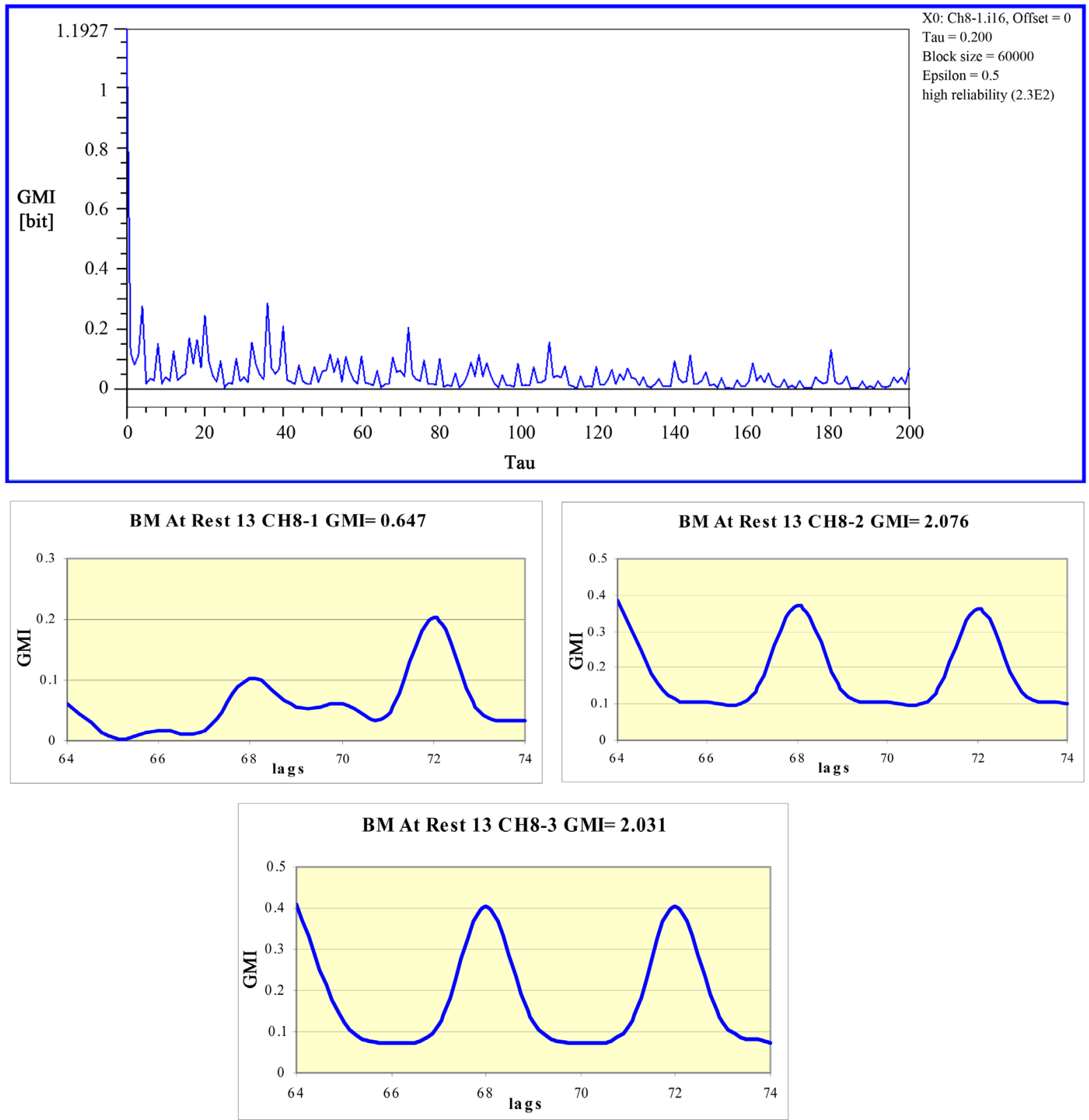

Figure 7. GMI analysis of the EEG in subject at rest affected from Facioscapulohumeral Muscular Dystrophy. 
ers in some time intervals but the significant result is that in order to recover in coherence, coupling, dependency and synchronization, the flow of information must increase largely. When we have time intervals of disfunction, the GMI value reaches the value of 0.647 Bit against the normal value ranging in 1.760 to $1.786 \mathrm{Bit}$ and, in order to recover, brain dynamics is forced to increase to GMI value until to $2.076 \mathrm{Bit}$.

Let us examine what it happens when the subject is performing the treatment. The results are in Figure 8.

For the whole set of the GMI we observe a net improvement. The whole coherence, dependency, flow of information in brain regions and synchronization evidence a net improvement with GMI values ranging now from 0.25 to 0.52 Bit. The SMR band gives a net recovery of GMI values but it remains still time intervals in which the GMI value is at so low GMI values. Peaks at the basic frequencies are recovered.

Let us examine now the results after the second session of the treatment at rest. The results are given in Figure 9.
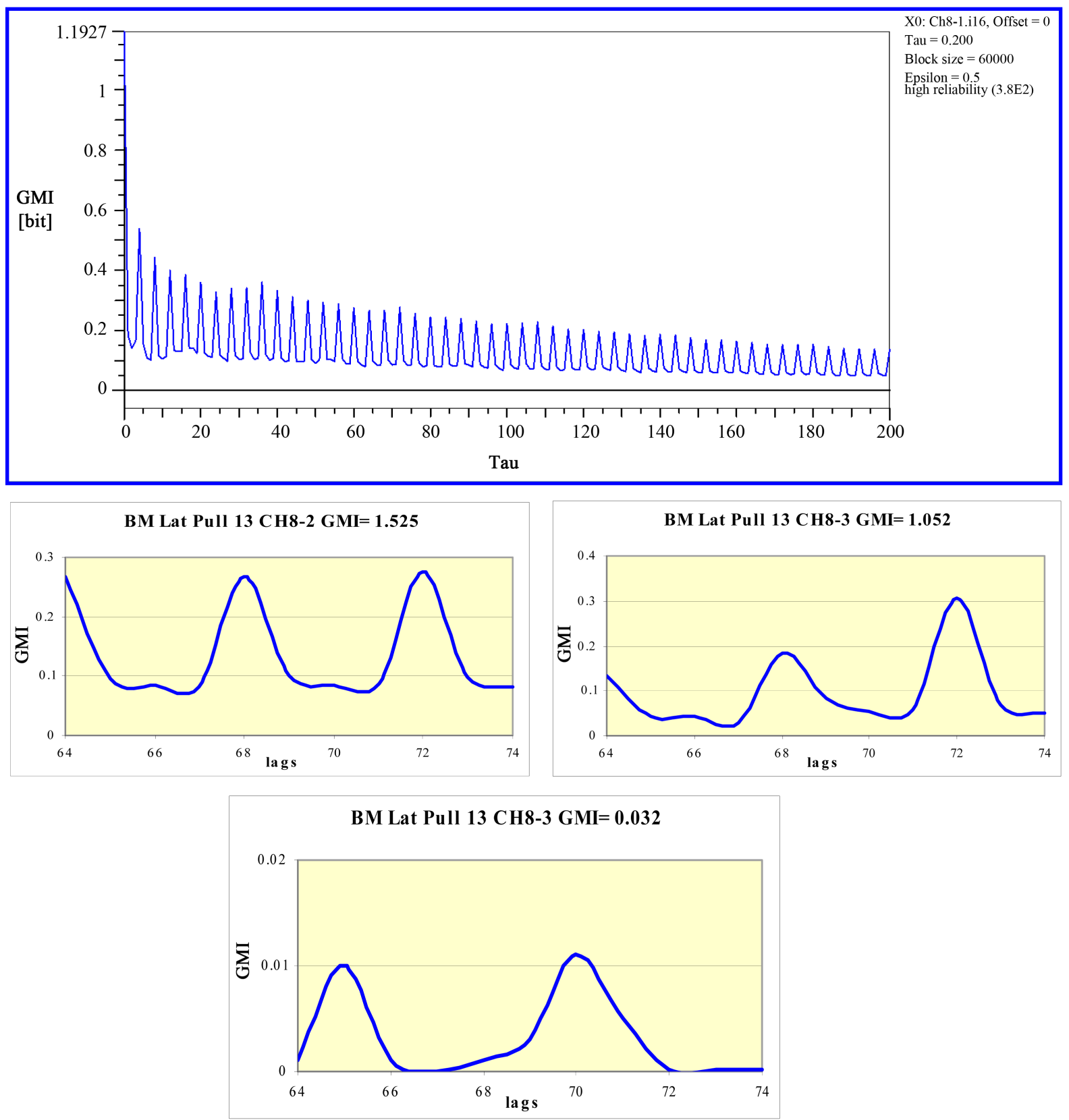

Figure 8. GMI results of the subject under first session of the treatment. 

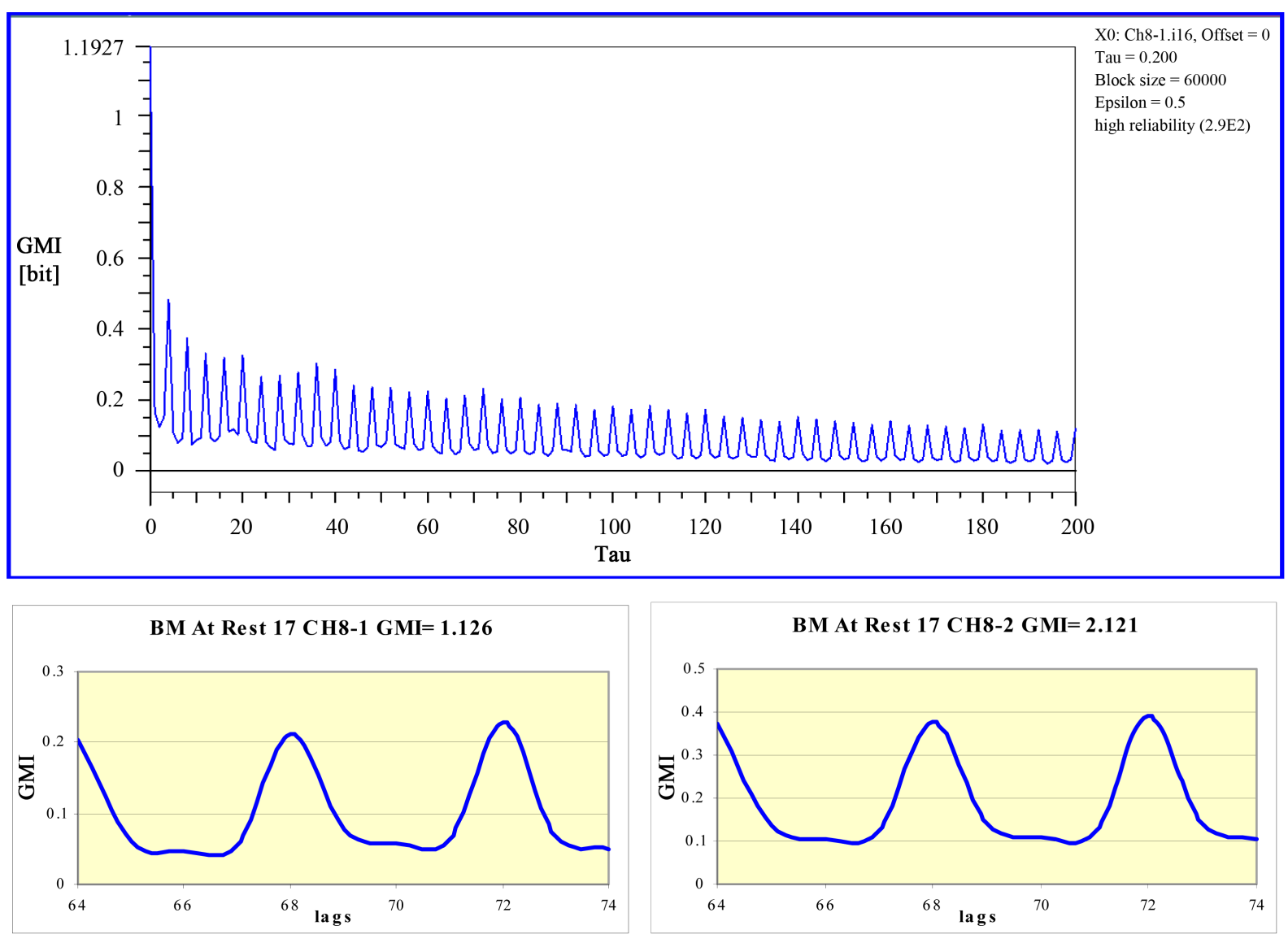

Figure 9. GMI results of the subject after the second session of the treatment.

Observing the results of Figure 9 we may conclude that the subject has reached a final satisfactory condition according to the GMI results. They are in accord with the net improvement had from the subject under clinical final evaluation. In the part II of this paper we will examine the results of the GMI in the coupling EMG-EEG.

\section{Conclusion}

In this paper we have given preliminary results about a case of Facioscapulohumeral Muscular Dystrophy after Ken Ware Neuro Physics Treatment. The finality of the study was to examine SMR brain dynamics of the subject at rest before the treatment and after the treatment and compare such analysis with the SMR brain dynamics of healthy subjects. Investigation of SMR band is to be considered a fundamental but difficult problem since the net SMR band is in principle rather complex to be isolated being the natural interference of such basic rhythm with alpha brain waves. In addition one has to acknowledge that the EMG signal originates actually from a strong non linear combination of similar distributions due to the single fiber action potential. This dynamics possibly determines a complex chaotic system. Consequently, all the standard methods, being all based on linear elaborations, cannot give accurate results since instead an analysis of the non linear and thus possibly chaotic systems is required using appropriate non linear methodologies. Our investigation is based on the use of the method of the Generalized Mutual Information since it enables to identify time dependencies, coupling, flow of information and thus synchronization within time series arising from a chaotic dynamics, accounting in detail for the contributions arising from the non linear acting mechanisms. The conclusion of the study seems promising since we have been able to identify in detail the SMR band dynamics and to use the GMI index of the Generalized Mutual Information to discriminate the control respect to the pathological condition. We have examined brain dynamics at time intervals of 1.04 milliseconds, thus investigating the time dynamics in great detail. The relevant result is that, in accord with the obtained GMI results, it seems that during movement brain reinforces its coherence, flow of information, dependencies in all brain interested regions and particularly in SMR band. 
This may be considered as an expected result but on the other hand we have to consider that GMI enables to quantify the level of brain coherence, synchronization, and dependency that is reached from brain during the movement and thus to give us important and accurate estimations of clinical importance. The possibility to quantify in detail the SMR activity during the movement assumes greater importance if we consider that usually, during EEG recording, the SMR band tends to decrease and such condition complicates the possibility of investigation by using traditional methods. The study reveals instead that, using GMI, we are able to quantify the effort of self-organization that brain performs during movement in relation to neurological activity that during this phase brain realizes. In addition, the study has given us the possibility of clinical evaluation since GMI has really discriminated between controls and the serious case of muscular dystrophy of the examined subject. The analysis has enabled us to characterize the serious initial pathological condition of the subject at starting and we have had also the possibility to follow the improvements of the subject during the treatment. The estimation of such results step by step has enabled us to conclude that, according to the GMI analysis, the subject receives a relevant improvement of his pathological condition as a consequence of Ken Ware Neuro Physics treatment. We have now in progress the analysis of the data by GMI crossing as well as EMG and EEG. We may anticipate that, according to our results, this analysis has revealed that the method is able to analyze in detail also such coupled dynamics with discriminating results between normal and pathological condition as well as during the phases of treatment.

\section{References}

[1] Pfurtscheller, G. (1999) EEG Event-Related Desynchronization (ERD) and Event-Related Synchronization (ERS). In: Niedermeyer, E. and Lopes da Silva, F.H., Eds., Electroencephalography: Basic Principles, Clinical Applications and Related Fields, 4th Edition, Williams and Wilkins, Baltimore, 958-967.

[2] Sterman, M.B. (1966) Physiological Origins and Functional Correlates of EEG Rhythmic Activities: Implications for Self-Regulation. Biofeedback and Self-Regulation, 21, 3-33.

[3] Sterman, M.B. and Wyrwicka, W. (1967) EEG Correlates of Sleep: Evidence for Separate Forebrain Substrates. Brain Research, 6, 143-163. http://dx.doi.org/10.1016/0006-8993(67)90186-2

[4] Sterman, M.B., Wyrwicka, W. and Roth, S.R. (1969) Electrophysiological Correlates and Neural Substrates of Alimentary Behavior in the Cat. Annals of the New York Academy of Science, 157, 723-739. http://dx.doi.org/10.1111/j.1749-6632.1969.tb12916.x

[5] Chase, M.H. and Harper, R.M. (1971) Somatomotor and Visceromotor Correlates of Operantly Conditioned 12 - $14 \mathrm{c} / \mathrm{s}$ Sensorimotor Cortical Activity. Electroencephalography and Clinical Neurophysiology, 31, 85-92. http://dx.doi.org/10.1016/0013-4694(71)90292-6

[6] Kumar, A., Agarwal, S., Agarwal, D. and Phadke, S.R. (2013) Myotonic Dystrophy Type 1 (DM1): A Triplet Repeat Expansion Disorder. Gene, 522, 226-302. http://dx.doi.org/10.1016/j.gene.2013.03.059

[7] Udd, B. and Krahe, R. (2012) The Myotonic Dystrophies: Molecular, Clinical, and Therapeutic Challenges. The Lancet Neurology, 11, 891-905. http://dx.doi.org/10.1016/S1474-4422(12)70204-1

[8] Angelini, C., Di Leo, R. and Cudia, P. (2013) Autonomic Regulation in Muscular Dystrophy. Frontiers in Physiology, 4, 1-4. http://dx.doi.org/10.3389/fphys.2013.00257

[9] Conte, E., Khrennikov, A.Y., Federici, A. and Zbilut, J.P. (2009) Fractal Fluctuations and Quantum-Like Chaos in the Brain by Analysis of Variability of Brain Waves: A New Method Based on a Fractal Variance Function and Random Matrix Theory: A Link with El Naschie Fractal Cantorian Space-Time and V. Weiss and H. Weiss Golden Ratio in Brain. Chaos, Solitons and Fractals, 41, 2790-2800. http://dx.doi.org/10.1016/j.chaos.2008.10.016

[10] Conte, E., Federici, A. and Zbilut, J.P. (2009) A New Method Based on Fractal Variance Function for Analysis and Quantification of Sympathetic and Vagal Activity in Variability of $R-R$ Time Series in ECG Signals. Chaos, Solitons and Fractals, 41, 1416-1426. http://dx.doi.org/10.1016/j.chaos.2008.05.025

[11] Conte, E., Pierri, G.P., Federici, A., Mendolicchio, L. and Zbilut, J.P. (2006) A Model of Biological Neuron with Terminal Chaos and Quantum-Like Features. Chaos, Solitons and Fractals, 30, 774-780. http://dx.doi.org/10.1016/j.chaos.2005.08.211

[12] Conte, E., Vena, A., Federici, A., Giuliani, R. and Zbilut, J.P. (2004) A Brief Note on Possible Detection of Physiological Singularities in Respiratory Dynamics by Recurrence Quantification Analysis of Lung Sounds. Chaos, Solitons and Fractals, 21, 869-877.

[13] Vena, A., Conte, E., Perchiazzi, G., Federici, A., Giuliani, R. and Zbilut, J.P. (2004) Detection of Physiological Singularities in Respiratory Dynamics Analyzed by Recurrence Quantification Analysis of Tracheal Sounds. Chaos, Solitons and Fractals, 22, 869-881. http://dx.doi.org/10.1016/j.chaos.2004.03.005 
[14] Conte, E., Todarello, O., Conte, S., Mendolicchio, L. and Federici, A. (2010) Methods and Applications of Non-Linear Analysis in Neurology and Psycho-Physiology. Journal of Consciousness Exploration and Research, 1, 1070-1138.

[15] Conte, E., Pieralice, M., Laterza, V., Losurdo, A., Santacroce, N., Conte, S., Federici, A. and Giuliani, A. (2012) Traditional and a New Methodology for Analysis of Heart Rate Variability: A Review by Physiological and Clinical Experimental Results. International Journal of Research and Review in Applied Sciences (IJRRAS), 13, 206-293.

[16] Pompe, B. (1993) Measuring Statistical Dependences in a Time Series. Journal of Statistical Physics, 73, 587-610. http://dx.doi.org/10.1007/BF01054341

[17] Ross, S.N. and Ware, K. (2013) Hypothesizing the Body's Genius to Trigger and Self-Organize Its Healing: 25 Years Using a Standardized Neurophysics Therapy. Frontiers in Physiology, 4, 1-18. http://dx.doi.org/10.3389/fphys.2013.00334 